\title{
Jörg Roesler
}

\section{Massenkonsum in der DDR: zwischen egalitä- rem Anspruch, Herrschaftslegitimation und „exquisiter" Individualisierung}

\section{Das Zeitalter des Massenkonsums: Für die DDR überhaupt ein Thema?}

Die Auffassung, dass das Zeitalter des Massenkonsums an der DDR wie auch den anderen realsozialistischen Ländern vorbeiging, ist kaum von der Hand zu weisen, solange man sich auf die Schlagworte der institutionell verankerten „Aufarbeitung der DDR-Geschichte“ verlässt. Demnach wurde der Bevölkerungskonsum über den Plan autoritär-diktatorisch geregelt. Die Konsumgüterindustrie hätte in der Gewichtung der Planziele stets hinten an gestanden. Der Schwerindustrie wurde aus ideologisch-politischen Gründen der Vorrang eingeräumt. Planwirtschaft war Befehlswirtschaft und auch der Konsum in Umfang und bis ins Detail zentralistisch vorgeschrieben. Das Ergebnis konnte daher nichts anderes sein als Mangel. Der Mangelgesellschaft im Osten habe die Überflussgesellschaft im Westen gegenüber gestanden. In der dichotomen Beschreibung der beiden deutschen Gesellschaften standen und stehen nicht nur Diktatur und Demokratie, nicht nur Plan- und Marktwirtschaft, sondern auch Massenkonsum und eine der Massen der Bevölkerung aufgezwungene Askese einander diametral gegenüber (Kaminsky 1999).

Doch entspricht dieses im Kalten Krieg geprägte und nach 1990 wieder belebte Bild der Realität von Konsumverhalten und Konsummöglichkeiten in der DDR? Bei näherem Hinsehen wird erkennbar, dass auch die Geschichte des Konsums in Ostdeutschland, im kämpferischen Ost-West-Diskurs damit stark vereinfacht wurde und immer noch wird.

Richtig ist allerdings zunächst einmal, dass die die Politik der DDR maßgeblich bestimmende SED-Führung sich die Konsumgesellschaft nicht als Ziel setzte. In Anknüpfung an Marx sah das im „allgemeinen Bewegungsgesetz der sozialistischen Produktionsweise" verankerte gesellschaftspolitische Ziel vor, den Menschen „die vollständige freie Ausbildung und Betätigung ihrer körperlichen und geistigen Anlagen“ zu ermöglichen. Die „ausreichende materielle Bedürfnisbefriedigung" galt dabei nur als Voraussetzung für die Verwirklichung dieses höheren Zieles (vgl. dazu MEW Bd. 20: 263). 
Die kommunistische Utopie schloss demnach die Konsumgesellschaft definitiv aus. In den daraus abgeleiteten Zielvorstellungen gab es keinen Platz für Produkt- bzw. Sortimentsvielfalt oder wechselnde Moden. Allerdings war die Zeit, in der eine Variante für verbindlich erklärt und die anderen verdammt wurden, in der DDR nur kurz. Beim Design z.B. reichte diese Zeit vom Herbst 1950 bis zum Herbst 1953. In dieser Zeit waren Abweichungen vom durch die SED akzeptierten Design - vor allem handelte es sich dabei um die „neue Sachlichkeit“ bzw. die Bauhaustradition - zu einer „volksfremden und volksfeindlichen Strömung“ erklärt worden (Bertsch u.a. 1991: 18). Doch mit dem konsumfreundlichen „Neuen Kurs“ konnten die Deutschen Werkstätten Hellerau, die einem Industrieministerium unterstellt waren, bei der Eröffnung ihres Berliner Industrieladens im Herbst 1953 Anbaumöbel anbieten, die bereits in den dreißiger Jahren als „wachsende Wohnung" konzipiert worden waren und einige Jahre nicht mehr produziert wurden, da ihre Formgebung ,in erster Linie aus der Funktion abgeleitet" gewesen war (Roesler 1995: 1137).

Im Unterschied zu der immer wieder von Pragmatismus gekennzeichneten Tagespolitik akzeptierte die kommunistische Utopie eine individuell unterschiedliche Nutzung der durch die Produktivitätsfortschritte im Kommunismus verfügbaren „disposal time“ explizit nur auf der Ebene der Befriedigung der „höheren“ Bedürfnisse. Außerhalb der angestrebten „Kulturgesellschaft“, auf der Ebene der materiellen Bedürfnisbefriedigung, wurde eher Gleichheit als Unterschiedlichkeit angestrebt. Deswegen wurde lohnpolitisch viel getan, damit sich die (Netto-)einkommen von Arbeitern und Angestellten wenig unterschieden. Das materielle Niveau, auf dem sich alle Schichten mehr oder minder treffen sollten, ist von der SED-Führung nie exakt bestimmt worden, lag aber zweifellos über dem Vorkriegsniveau der Arbeiter und unter dem der „bürgerlichen“ Schichten (Wilczek 2004: 214f.).

Für die dem kommunistischen Stadium vorgelagerte sozialistische Periode - in der sich die DDR nach Auffassung der SED-Führung seit Beginn der 60er Jahre befand - sollte es allerdings noch gewisse Differenzierungen nach der „Arbeitsleistung" geben, die über eine unterschiedliche Entlohnung auch zu Unterschieden im Konsumgüterverbrauch führen mussten. Jedoch sollten die Differenzen gering sein und auf keinen Fall die Herstellung von „Luxusgütern“ für eine bestimmte Käuferschicht rechtfertigen, zumal soziale Distinktion in der propagierten „sozialistischen Menschengemeinschaft“ als Fehl am Platze galt.

Im Gegensatz zum Konsumziel wurde das Produktivitätsziel des Kapitalismus und damit auch die fordistische Produktionsweise ohne Abstriche aus dem Kapitalismus übernommen. Nur schneller als im Westen sollte in Osteuropa wegen des historischen begründeten Rückstandes in der Technologie - die Produktivität wachsen. Diese Vorgabe galt auch für die DDR, deren technologischer Rückstand nicht historisch begründet, deren Produktivität aber durch 
das außerordentliche Ausmaß der sowjetischen Demontagen in der Nachkriegszeit um Jahrzehnte zurückgeworfen worden war. Das Aufholen schien prinzipiell möglich, schon weil ein Teil der im Westen beim Aufbau einer üppigen Konsumwelt verbrauchten Investitionen im Osten zusätzlich für den Aufbau moderner Produktionsanlagen verwendet werden konnte.

Für die dem Kommunismus vorangehende (real)sozialistische Phase galt es daher ein „Gesetz des vorrangigen Wachstums der Produktion von Produktionsmitteln" zu befolgen, das - grob gesehen - der Schwerindustrie gegenüber der Konsumgüter produzierenden „Leichtindustrie“ ein Primat zuordnete. Die „Leichtindustrie“ war damit jedoch nicht an den Rand gedrängt. Auch noch Mitte der 80er Jahre war nach sorgfältigen Berechnungen seitens des Deutschen Instituts für Wirtschaftsforschung (DIW) ein Viertel der in der DDRIndustrie Beschäftigten in der Konsumgüterproduktion eingesetzt. Immerhin 21\% stellten als Finalprodukt Konsumgüter her (Cornelsen u.a. 1985: 302f). Der Anteil überwiegend Konsumgüter herstellender Bereiche am Bruttoanlagevermögen der DDR belief sich 1983 in der DDR auf 30,5\%, in der Bundesrepublik zur gleichen Zeit etwa auf 31, 5\% (Materialien 1987: 293).

So erweist sich die These von der Dominanz der Schwerindustrie als stark übertrieben, ebenso jene von der strengen Zentralisierung, nach der die zu produzierenden Konsumgüter in Umfang und Sortiment dem Konsumenten vorgegeben wurden. In der Konsumplanung galt ab Mitte der 50er Jahre für die Betriebe keineswegs die Devise „Friss Vogel oder stirb!“. In einer Studie des DIW über die Konsumgüterplanung in der DDR Mitte der 80er Jahre hieß es zum Planungsprozess:

„Die Konsumgüterplanung verläuft als wiederholter Informationsaustausch- und Korrekturprozess, der in horizontaler und vertikaler Richtung verflochten ist. Die zentralen Entscheidungen beginnen mit der Festlegung gesamtwirtschaftlicher Proportionen (Akkumulation und Konsumtion, Kauffonds und Warenfonds) und setzten sich in einem komplizierten Bilanzierungsprozess bis zu detaillierten Warengruppen und Sortimenten fort. An diesem Entscheidungsprozess sind verschiedene Entscheidungsebenen von der Partei- und Wirtschaftsführung bis zu Produktion und Handel beteiligt." (Cornelsen u. a. 1985: 27f)

Wenn auch dieses Verfahren keineswegs garantieren konnte, dass immer entsprechend dem Bedarf produziert wurde, so sicherte es doch die Annäherung an die realen Konsumtionsbedürfnisse der Bevölkerung.

Das von den „Aufarbeitern der DDR-Geschichte“ geprägte Bild von der ostdeutschen Mangelgesellschaft im Gegensatz zur westdeutschen Überflussgesellschaft verliert weiter an Glaubwürdigkeit, wenn man die Aussagen zum Stand der Versorgung mit Lebensmitteln und zur Ausstattung mit gewerblichen Konsumgütern für die DDR und die BRD zur Kenntnis nimmt, wie sie für den Bericht des Bundeskanzlers „Zur Lage der Nation im geteilten Deutschland“ im Jahre 1987 von führenden bundesdeutschen Wirtschaftsinstituten erarbeitet wurden. Danach war „die Ernährung in beiden deutschen Staaten durch eine überhöhte Zufuhr von Nährstoffen gekennzeichnet. So lag in der 
DDR der durchschnittliche energetische Verbrauch der Bevölkerung 1980 mit einem Viertel über der Norm der DDR-Ernährungswissenschaft; mindestens jeder vierte Erwachsene gilt heute als übergewichtig." Und der Bericht fügt hinzu: „Ähnliches wird für die Bundesrepublik festgestellt.“ Die festgestellte Ähnlichkeit wurde nicht nur auf der „All you can eat“-Ebene festgestellt. „Bei vielen Konsumgütern im Bereich der Haushaltsführung ist sowohl in der Bundesrepublik als auch in der DDR ein hoher Ausstattungsgrad zu konstatieren, so bei Kühlschränken, Waschmaschinen, Staubsaugern, elektrischen Handrührern oder -mixern und elektrischen Kaffeemühlen; teilweise kann von Vollausstattung gesprochen werden" (Materialien 1987: 525, 529).

Unzureichende Bedürfnisbefriedigung, ja direkten Mangel an Konsumgütern hat es, worauf noch einzugehen sind wird, in der DDR ungeachtet dessen gegeben. Aber generell war der Mangel - was bei der Verwendung des Labels „Mangelgesellschaft“ rasch in Vergessenheit gerät - relativ, nicht absolut.

Für die Einbeziehung der DDR-Gesellschaft in die Kategorie Konsumgesellschaft spricht, ungeachtet der anders gearteten, aus der kommunistischen Utopie abgeleiteten Zielvorstellungen, einiges. Das wurde vereinzelt auch recht früh erkannt (Sywottek 1993: 271ff). Zu klären bleibt aber ein offensichtlicher Widerspruch: Wie konnten bei deutlich unterschiedlichen konsumstrategischen Zielsetzungen in Ost und West doch einander ähnelnde Ergebnisse zustande kommen?

\section{Konsumziele, Konsumwünsche und ein notwendiges Arrangement}

Die Antwort für die DDR liegt offensichtlich bei den Konsumenten, deren Auffassungen von Konsum bisher noch nicht in die Betrachtung einbezogen wurden. Ihre Ansprüche waren nicht mit denen der SED-Führung identisch. Teilweise stimmten sie mit den Zielstellungen der kommunistischen Utopie überein. Das Streben nach sozialer Gleichheit war in der Arbeiterschaft, die zusammen mit den „kleinen“ Angestellten etwa drei Viertel der Bevölkerung der DDR ausmachte, traditionell weit verbreitet. Die Forderung, den Verbrauch auf ein „vernünftiges Maß“ zu beschränken - Konsumgüter sollten sich durch Langlebigkeit und entsprechende Gebrauchswerteigenschaften auszeichnen - war in der konsumgenossenschaftlichen Tradition der Arbeiter verankert. Wirtschaftskrise und zweiter Weltkrieg, die die Erfahrungen der Mehrzahl der DDR-Bewohner der 50er Jahre geprägt hatten, haben diese Einstellung zweifellos gefestigt. Die führenden SED-Vertreter, die ähnliche Erfahrungen auch unmittelbar persönlich gewonnen und verarbeitet hatten, fühlten sich insoweit - nicht zu unrecht - eins mit der Arbeiterschaft.

Von der Führung zu wenig bedacht worden war jedoch, dass von der proletarischen Bevölkerung aus der Not eine Tugend gemacht worden war, und dass

1 Ausgewiesen wurde in der DDR-Statistik nur der Anteil der Arbeiter und Angestellten insgesamt. Bezogen auf alle Berufstätigen lag er 1960 bei 80,6 \% und 1989 bei 88,2 \%. 
sich die Unterschichten der bürgerlichen Gesellschaft in ihren Konsumwünschen und -sehnsüchten schon immer an den Lebensstilen der bürgerlichen Oberschicht orientiert hatten. Reste dieser Oberschichten gab es auch in der DDR noch - Kleinkapitalisten und Handwerksmeister. Ihr Anteil an der erwerbstätigen Bevölkerung der DDR sank zwischen 1950 und 1983 von 18\% auf 2\%, ihr Anteil an den „Geldeinnahmen aus Erwerbstätigkeit (netto)“ im gleichen Zeitraum von 24\% auf 6\% (Vortmann 1985: 136, 179f). Vor allem in den Klein- und Mittelstädten blieben sie, soweit es den Konsumgeschmack betraf, oftmals beneidete Trendsetter (Merkel 2004: 16). ${ }^{2}$ Wichtiger in diesem Zusammenhang war jedoch spätestens mit der größeren Verbreitung des Fernsehens in der DDR seit der zweiten Hälfte der 50er Jahre der Einfluss der Konsumwelt der Westdeutschen. So bot das „Wirtschaftswunder“ in Westdeutschland den meisten Angehörigen auch der unteren Schichten Zugang zu einer verheißungsvollen Konsumwelt, sofern sie die harschen Bedingungen der Akkordlohnarbeit akzeptierten (Schmiede/Schudlich 1977: 322 ff).

In der DDR hatten die „Werktätigen“ nach Meinung der SED-Führung eine derartige materielle Tröstung nicht nötig. Ihre Arbeit galt als „befreite Arbeit“ und die Heldentaten der Aktivisten sowie die jährlichen Wettbewerbsinitiativen zur Erfüllung und Übererfüllung der Pläne waren der immer wieder zitierte Nachweis, dass die Mehrzahl der Arbeiter den „neuen Charakter der Arbeit“ im Sozialismus auch bereits erkannt habe (Falk/Barthel 1966: 9ff). Tatsächlich blieben derartige Auffassungen, die mit Hilfe der sich seit Beginn der 50er Jahre rasch ausbreitenden Brigadebewegung an alle Arbeiter und Angestellten Ostdeutschlands herangetragen werden sollten, die Meinung einer engagierten Minderheit. Die Mehrheit der Arbeiter sah ihre Arbeitsbedingungen so wie sie waren: fremdbestimmt (im weiterhin fordistisch organisierten Betrieb). Dass der Akkordlohn nunmehr offiziell Leistungslohn hieß, machte ihn nicht besser (Roesler 1997: 5ff). Die Arbeit im „volkseigenen Betrieb“ konnte deshalb für die Mehrheit der Werktätigen kein Ersatz sein für den Eintritt in jene Konsumwelt, die sich ihren westdeutschen „Klassengenossen“ im Tauschhandel durch die Akzeptanz eines Höchstmaßes an kontrollierter Arbeit zu erschließen begann - sie forderten sie auch ein.

$\mathrm{Da}$ es der SED-Führung nicht gelang, sich mit dem „neuen Charakter der Arbeit“ gegenüber der Masse der Werktätigen zu legitimieren, musste auch sie zur Loyalitätssicherung jenem Tauschhandel zustimmen und ihren Untertanen die Aussicht auf die neue Konsumwelt erschließen.

2 Nach Berechnungen von Ina Merkel lag das Einkommen der Handwerker und der Gewerbetreibenden in der DDR 1970 beim 1,5 bis 1,7fachen des durchschnittlichen Nettoeinkommens der DDR-Bürger. Bei der Vorbereitung auf die Verstaatlichung der privaten Unternehmen und gut verdienenden Handwerkergenossenschaften benutzte die SED-Führung den Sozialneid der Arbeiter und kleinen Angestellten, um die Unternehmer (und die ihre Interessen vertretenden Blockparteien) gefügig zu machen. 
Ein Kompromiss zwischen den aus der kommunistischen Utopie abgeleiteten Zielvorstellungen und jenen, die sich die Mehrheit der Arbeiter selbst gab, musste gefunden werden. Die SED-Führung war gezwungen, sich zu arrangieren. Sie sah in der Ausrichtung der DDR-Gesellschaft auf Massenkonsum deutlich Risiko und Chance. Das Risiko bestand, solange die DDR für die Sowjetunion unverzichtbar war, kaum darin, dass bei allzu großer Imitation des Westens den Arbeitern das Original zu nahe gebracht worden wäre. Es bestand darin, dass durch allzu großes Nachgeben gegenüber den „ordinären“ Konsumentenwünschen die entsprechenden Investitionen die angestrebte wirtschaftliche Überlegenheit über das kapitalistische System gefährden könnten. Die Chance bestand darin, durch eine auf die Wünsche der Masse der Bevölkerung eingehende Konsumpolitik eine breite Zustimmung auch für die auf die Verwirklichung der kommunistischen Utopie gerichteten Ziele zu erwerben. Ein derartiges Denken war der SED-Führung, die ursprünglich von der Attraktivität ihrer Utopie für „breite Volksmassen“ überzeugt war, zunächst nicht eigen. Jedoch lehrten sie die Erfahrungen der 50er Jahre, dass es weniger die Ideologie war, weder die eigene, noch die gegnerische, die die Massen bewegte, als der Konsum. Nachweisen lässt sich das an der „Republikflucht“, eine Migrationsbewegung von Ost nach West, an der mehrheitlich Arbeiterfamilien teilnahmen. „Die Mehrheit derjenigen, die die Deutsche Demokratische Republik verlassen,“ wurde auf einer internen Sitzung der von der SEDFührung eingesetzten „Kommission zu den Fragen der Republikflucht“ im November 1956, auf dem Höhepunkt der Ost-West-Wanderung, unumwunden zugegeben, „tun das nicht deshalb, weil sie mit unserer Ordnung nicht einverstanden sind, sondern vor allem aus ökonomischen und anderen Ursachen heraus" (Major 2000: 223). Folgerichtig beließ es die SED-Führung im Kampf gegen die „Abwerbung“ nicht bei polizeilichen Maßnahmen, sondern Ulbricht entwickelte auf dem V. Parteitag der SED für die Zeit des Siebenjahrplanes (1959-1965), gestützt u.a. durch Chruschtschows Lieferzusagen für die DDR als „Schaufenster des Sozialismus“ in Europa, die „ökonomische Hauptaufgabe“, die darin bestehen sollte, die Volkswirtschaft der DDR ,innerhalb weniger Jahre so zu entwickeln, dass der Pro-Kopf-Verbrauch unserer Werktätigen Bevölkerung mit allen wichtigen Lebensmitteln und Konsumgütern den ProKopf-Verbrauch der Gesamtbevölkerung (Hervorhebung J.R.) in Westdeutschland erreicht und übertrifft" (Ulbricht 1959: 18). Die Wirkung des mit einer Reihe von Sofortmaßnahmen (Lohnerhöhungen, Abschaffung der Lebensmittelkarten) unterstützten Versprechens war eindeutig. Die Zahl der Ost-WestMigranten halbierte sich 1959 gegenüber 1957 und stieg erst im 1961 wieder an (Storbeck 1963: 154), als sich die Ziele des Siebenjahrplanes als allzu hoch gesteckt erwiesen und erneut Versorgungsengpässe auftraten. Im Mai hatte Ulbricht bereits intern zu verstehen gegeben, „dass nach seiner Einschätzung die Republikflucht zunehmen wird“ (Steiner 2004: 120). Verschärft wurde die Ver- 
sorgungskrise von 1961 durch die Zwangskollektivierung vom Frühjahr 1960, über die Ulbricht die Voraussetzung für die „Angleichung der Lebensbedingungen in Stadt und Land" zu schaffen gedachte - einem wesentlichen Bestandteil der aus der kommunistischen Utopie abgeleiteten Gleichheitsforderungen (Geschichte der SED 1978: 400ff).

Wie dieses in sich widersprüchliche konsumpolitische Zielbündel konkret aussah und in welchem Maße es unter wechselnden wirtschaftlichen Bedingungen realisiert werden konnte, ist den folgenden drei Abschnitten zu entnehmen. Dabei kann, anders als dies für die Entwicklung in der Bundesrepublik vielleicht möglich ist, die Entwicklung des Massenkonsums in Ostdeutschland nicht unter Ausblendung der wirtschaftlichen Rahmenbedingungen und ökonomischen Strukturen der DDR analysiert werden.

\section{Bemühungen um die planmäßige Gestaltung eines sozialistischen Massenkonsums in den 1960er Jahren}

Die Überlegungen, auf welche Weise die Konzentration auf die Entwicklung der Produktivkräfte einerseits und auf die Bereitstellung von Gütern für den Massenkonsum andererseits im Plan sinnvoll vereint werden konnten, standen zu Beginn der 60er Jahre unter dem Eindruck eines Fehlschlages. Im 1959 beschlossenen Siebenjahrplan hatte sich die SED-Führung um die Verträglichkeit der widersprüchlichen Zielstellungen wenig Gedanken gemacht und beschlossen „durch Erhöhung der Arbeitsproduktivität und Steigerung der Produktion Westdeutschland im Pro-Kopf-Verbrauch bei den meisten industriellen Konsumgütern und Lebensmitteln bis Ende 1961 einzuholen und zu überholen“ (Siebenjahrplan 1959: 159). Bereits vor Ablauf des selbst gesetzten Zeitlimits war deutlich geworden, dass das angestrebte doppelte Ziel „in historisch kürzester Frist“ zu erreichen, unrealistisch war. Die Steigerung des Umfangs und die Erhöhung der Produktivität der fordistischen Produktion in der DDR konnte, so die Schlussfolgerung der SED-Führung aus der Wirtschaftskrise zu Beginn der 60er Jahre, nicht durch eine einmalige Kraftanstrengung, sondern nur durch beständige Produktivitätserhöhungen als Resultat der Freisetzung schöpferischer Kräfte bei Managern und Arbeitern auf der Grundlage der Dezentralisierung der Planentscheidungen erreicht werden. So glaubte man die materiellen Voraussetzungen schaffen zu können, um die von den „Werktätigen“ erwartete Steigerung des Konsums zu verwirklichen ohne die Modernisierung und den Neubau von Industrieanlagen zu gefährden. Das Eingehen auf die Konsumwünsche der Masse der Bevölkerung sollte nicht zu einer Konsumgesellschaft wie im Westen führen, wohl aber an deren „Errungenschaften“ anknüpfen, ohne die entsprechenden Ziele der kommunistischen Utopie aufzugeben.

Um für den sozialistischen Massenkonsum die notwendige Akzeptanz zu schaffen und ihn planerisch zu bewältigen, wurden zwei bereits vorhandene 
Instrumente der Konsumlenkung weiterentwickelt: Bedarfsforschung und Konsumwerbung. Aufgabe der Konsumforschung, die ab 1962 vom „Institut für Bedarfsforschung“ (ab 1967 „Institut für Marktforschung“) betrieben wurde, war es, exakte Vorhersagen darüber zu machen, welche Konsumgüter die Bevölkerung in welchen Mengen benötige. Damit sollten „Engpässe“ auf der einen und „Überplanbestände“ auf der anderen Seite, sichtbares Zeichen, dass an den Bedürfnissen der Bevölkerung vorbei produziert worden war, vermieden werden. Zu diesem Zweck erhielt das Marktforschungsinstitut als erste und über einen beträchtlichen Zeitraum einzige Einrichtung der DDR die Möglichkeit, repräsentative Befragungen der Bevölkerung vorzunehmen. Es ging der Bedarfsforschung aber nicht nur darum, die Konsumwünsche der Arbeiter und Angestellten in Erfahrung zu bringen. Auf die Vorstellungen der „Werktätigen“ sollte auch gezielt im Sinne dessen, was an Verbrauch unter sozialistischen Bedingungen als rational und sinnvoll erachtet wurde, Einfluss genommen werden. Es sollten nicht nur Bedürfnisse erforscht, sondern auch an- bzw. aberzogen werden (Cornelsen u.a.: 12ff). In diesem Zusammenhang kam dem zweiten der beiden Instrumente, der Konsumwerbung eine wichtige Rolle zu. Die Konsumwerbung der DDR erhielt erstens die Aufgabe, jene (neuen) Konsumgüter zu propagieren, die von der eigenen Industrie in ausreichender Menge und kostengünstig hergestellt werden konnten. Wurden diese Waren unermüdlich angepriesen, so durfte für Güter, bei denen der Bevölkerungsbedarf das Aufkommen überstieg, prinzipiell nicht geworben werden. Begrenzt war z.B. in den 60er Jahren das Angebot an Butter. Das hatte seine Ursache in den durch die vollständige Kollektivierung der Landwirtschaft 1960 bedingten Strukturumbrüchen, deren Auswirkungen auf die tierische Produktion bis Ende der 60er Jahre spürbar waren. Ausreichend Rohstoffe und Kapazitäten standen in der DDR dagegen für die Margarineproduktion zur Verfügung. Mit der Propagierung von „Goldina butterfein“, aus „bekömmlichem Sonnenblumenöl mit frischer Vollmilch verkirnt, vitaminreich, appetitlich, nahrhaft und delikat" wurde versucht, dem Verbraucher einen vollwertigen Ersatz für Butter zu suggerieren, um damit einen Engpass zu beseitigen und zugleich die vollständige Kapazitätsauslastung in der Öl- und Margarineindustrie zu sichern (Roesler 1988: 40ff).

Eine zweite Aufgabe der DDR-Werbung bestand darin, die Auffassung, dass der Konsum auf das „eigentlich Notwendige“ zu beschränken sei, zu bestärken, was u.a. hieß, eine „westliche“ Sortiments- und Produktvielfalt nicht zuzulassen. Bei vielen Gütern stimmten während der 1950er Jahre traditionelle Sparvorstellungen und die Konsumpolitik der SED-Führung noch überein. Schwerer zu vermitteln war diese Auffassung ab Ende der 50er, Anfang der 60er Jahre, als die „Beschränkung auf den rationalen Verbrauch“ mit der von der DDR-Bevölkerung zunehmend geforderten, weil „im Westen“ inzwischen für jedermann verfügbaren, modischen Vielfalt kollidierte. Hatte das 1952 gegründete DDR-Modeinstitut in den 50er Jahren noch versucht, die westliche 
Mode als „Dekadenz oder Kitschproduktion“ abzuqualifizieren, sie als „Mode um des Profits willen“ bzw. „Mode um der Mode willen“ abzustempeln und den „hektischen Modewechsel“ kritisiert, so sah das Modeinstitut sich Anfang der 60er Jahre gezwungen, seine Strategie zu ändern: „Keine absolute Ignorierung des kapitalistischen Modeschaffens“ war mehr zu propagieren, „vielmehr eine kritische Auswertung" westlicher Modetrends vorzunehmen. Die Mode der DDR sollte nunmehr ,neben der Berücksichtigung der nationalen Traditionen des deutschen Volkes, des Standes der gesellschaftlichen Entwicklung in unserem Arbeiter- und Bauernstaat und der ökonomischen Bedingungen“", d.h. der Produktionsmöglichkeiten und Importaufwendungen - J. R.) auch dem „Weltstand der internationalen Mode“ Beachtung zu zollen. Nicht Geltungskonsum im Modebereich aber doch eine gewisse Modevielfalt zuzulassen, war nunmehr die Devise. Auf diese Weise glaubte das Modeinstitut den „richtigen marxistischen Weg des Modeschaffens“ für die 60er Jahre gefunden zu haben (Deutsches Modeinstitut 1960, unpaginiert).

Für eine Reihe von „industriellen Konsumgütern“, deren in der zweiten Hälfte der 50er Jahre aufgenommene Produktion relativ aufwändig war, propagierten die Institutionen zur Konsumlenkung in der DDR deren kollektive Nutzung in deutlicher Abgrenzung zu der auf das zahlungskräftige Individuum zielende Konsumwerbung im Westen. Deshalb wurde in den 60er Jahren zunächst versucht, dem individuellen Kauf von modernen Haushalts- und Freizeitgeräten durch die Schaffung öffentlicher Ausleihmöglichkeiten gegenzusteuern. Das betraf Staubsauger, Fernseher, Fotoapparate, aber auch Sport- und Campingartikel. Offensichtlich nicht auf diese Art „vergesellschaften“ ließen sich Pkws. Ihr privater Besitz galt auch in den 60er Jahren weiterhin als Luxus. Im Jahre 1960 verfügte erst jeder 31. DDR-Haushalt über einen PKW Marke „Wartburg“ oder „Trabant“, 1965 jeder 12. und 1970 jeder sechste (Mittelbach/Roesler 1989: 199). Zum gleichen Zeitpunkt besaß in der Bundesrepublik bereits etwa jeder zweite private Haushalt einen PKW.

Die Vorgaben zur Marktregulierung durch die DDR-Institutionen für die Planung und Lenkung des Bevölkerungskonsums, die Aufklärungsarbeit der Werbung über akzeptable und über nicht zu akzeptierende Konsumwünsche waren nur teilweise von Erfolg gekrönt. So stieg einerseits der Margarineverbrauch mit „Goldina, „Sahna“, „Diät“, „Marina“ und „Sonja“ war inzwischen eine gewisse Markenvielfalt erreicht worden - in der zweiten Hälfte der 60er Jahre an. Der Butterverbrauch ging zurück (Roesler 1988: 45f). Die kollektive Nutzung von produktionsaufwändigen Haushaltsgeräten setzte sich andererseits nicht durch. Hier musste dem Streben der Bevölkerung nach individuellem Gebrauch und Besitz nachgegeben werden. Zwischen 1960 und 1970 erhöhte sich die Zahl der Haushalte, die über Waschmaschinen verfügten, von 6\% auf 56\%, bei Fernsehern von 19\% auf 76\% von Kühlschränken von $6 \%$ auf 56\%. (Mittelbach/Roesler 1989: 199). 
Zur Unzufriedenheit gerade der Arbeiterschaft über die Versorgung kam es 1969/70 jedoch nicht, weil die SED-Führung sich weitergehenden Konsumwünschen der Bevölkerung abgeneigt zeigte, sondern weil bereits zugesagte und zeitweise auch eingehaltene Konsumversprechen Ende der 1960er Jahre nicht mehr erfüllt wurden. Die Ursache war eine 1967/68 eingeleitete Kampagne der SED-Führung, die unter dem Slogan „Überholen ohne einzuholen“ eine erhebliche Beschleunigung der technologischen Modernisierung der Industriestruktur der DDR zu verwirklichen begann. Überholt werden sollte die Bundesrepublik zunächst einmal im Bereich von Wissenschaft, Technik und Produktivität. Die mit dieser Kampagne verbundene Umschichtung der Investitionen zugunsten der Industrieanlagen herstellenden Branchen führte bei begrenzter Investitionskapazität bald zu Einschränkungen bei der Produktion von Konsumgütern und damit auch zu - bereits überwunden geglaubten Knappheiten in der Versorgung. Die Unzufriedenheit der Bevölkerung über „Rhythmusstörungen“ im Konsumgüterangebot machte sich Ulbrichts designierter Nachfolger Honecker zunutze, um jenen zu stürzen und selbst die Macht zu übernehmen. Wenn sich der Machtwechsel mit Unterstützung durch die sowjetische Führung auch ohne Komplikationen vollzog, so hatte Honecker doch die Frage zu beantworten, wie er Ulbrichts Problem - gleichzeitig technologisch mit dem Westen mitzuhalten und eine Steigerung des Massenkonsums zu gewährleisten - zu lösen gedachte.

\section{Stärker am Westen orientierte Entwicklung des Massenkonsums in der DDR in den 1970er Jahren}

Gestützt auf die seit Anfang der 70er Jahre bei den internationalen Großbanken vorhandene Bereitschaft, den realsozialistischen Ländern Kredite größeren Umfangs zu gewähren, entwickelte die DDR unter Honecker eine Strategie der importinduzierten technologischen Modernisierung. Die Absicht war folgende: Die DDR importiert moderne Technik bis hin zur schlüsselfertigen Fabrik. Die zusätzlich entstehenden Produktionsstätten werden von vornherein so konzipiert, das nach deren Fertigstellung ein beträchtlicher Teil ihrer Produktion - mit neuester Technik erzeugte, modernen Standards entsprechende Produkte - auf westlichen Märkten abgesetzt werden können. Mit Hilfe der Deviseneinnahmen aus dem zusätzlichen Westexport würde es dann innerhalb von einigen Jahren möglich sein, die für die Fabrik oder den Kauf von Einzelmaschinen verauslagten Dollars oder DM zurückzuzahlen. Der Schuldenaufnahme würde die Entschuldung - zeitverzögert - auf dem Fuße folgen. Mit dieser Strategie glaubte die SED-Führung sich den nötigen Spielraum geschaffen zu haben, um einerseits gegenüber dem Westen technologisch wettbewerbsfähig zu bleiben und andererseits über die Mittel zu verfügen, stärker auf die Konsumwünsche der Bevölkerung einzugehen. Mit ihr die Übereinstim- 
mung zu suchen, empfahl sich für Honecker nicht nur aus prinzipiellen Legitimationsgründen, sondern auch, um sich selbst gegenüber seinem Vorgänger bei der Arbeiterschaft in ein günstiges Licht setzen zu können.

Bei dieser Zielsetzung war verständlich, dass im Rahmen der neuen Strategie nicht nur Produktionsmittel, sondern auch westliche Konsumgüter direkt importiert wurden. Der Anteil von Verbrauchsgütern sowie Textilien und Bekleidung an den Einfuhren der DDR aus westlichen Industrieländern (ohne Bundesrepublik) erhöhte sich im Zeitraum 1971-1975 gegenüber den vorangegangenen fünf Jahren von 7,5\% auf 12,7\%. Er hatte seinen Höhepunkt 1973 mit 17,4\% erreicht (DIW 1977: 357). Wenn diese zusätzlichen Konsumgüterimporte in ihrem Umfang auch nicht überschätzt werden dürfen - mit Westimporten, von Bananen bis Jeans, konnte Honecker der DDR-Bevölkerung anschaulich die Vorteile seiner Strategie der „Einheit von Wirtschafts- und Sozialpolitik" demonstrieren.

Unter den in den ersten Jahren relativ günstigen, aber dann seitens der SEDFührung viel zu lange weiterhin als günstig betrachteten finanziellen und wirtschaftlichen Bedingungen wurden eine Reihe von Grundsätzen des „sozialistischen Massenkonsums“, die in den 1960er Jahren gegolten hatten, aufgegeben. Das betraf sowohl die bisher gepflegte Auffassung vom PKW als Luxusgut als auch die vom Deutschen Modeinstitut (der DDR) vertretene Abneigung gegenüber der Produktvielfalt, wie sie der Westen mit der Werbung für unterschiedliche Marken demonstrierte.

Was den Besitz von privaten PKW betraf, so stieg dieser während der 1970er Jahre in der DDR deutlich an. Verfügte 1970 erst jeder 6. Haushalt in der DDR über einen PKW, so waren es 1980 bereits zwei von fünf. Die Lockerung der Vorgaben über den „sozialistischen Massenkonsum“ bis zum teilweisen Verzicht darauf nahm der DDR-Werbung einen wesentlichen Teil ihrer Funktion. Wenn auch in den 1970er Jahren Levis oder PKW der Marke „Golf“ nur als „Extras“ importiert wurden, so bedurften diese doch im Osten keiner Werbung, um von der Bevölkerung angenommen zu werden. Die war bereits im Westen „gelaufen“ und dank der umfassenden Ausstattung der DDR-Haushalte mit Fernsehgeräten war sie im Osten auch wahrgenommen worden. Im Jahre 1975 verabschiedete der Ministerrat der DDR dann auch folgerichtig eine Anordnung, die praktisch ein Werbeverbot für Konsumgüter bedeutete. Gestattet war nur noch die Werbung für Materialökonomie, Gesundheitsschutz, Versicherung, Kulturpolitik, für Produktionspropaganda und für das Lotto (Merkel 2004: 15). Werbung für Konsumgüter reduzierte sich auf Schaufensterwerbung. Auch diese wurde vielfach vernachlässigt (Schmidt 1994: 364ff).

Zumindest was die Quantität betraf, hatte sich - gemessen am Ausstattungsgrad der Haushalte - in der DDR bis Ende der 1970er Jahre bei den meisten Lebensmitteln (mit Ausnahme von Südfrüchten) und bei einem Großteil der 
technisierten Haushaltsgeräte eine stete Annäherung an das bundesdeutsche Niveau ergeben. Seitens der SED-Führung wurde zwar nicht mehr die Politik verfolgt, in einem bestimmten Zeitraum die Bundesrepublik im Konsum- und Produktivitätsniveau einzuholen oder gar zu überholen, doch hielt sie an der kontinuierlichen Verbesserung des Massenkonsums und seiner stärkeren Ausrichtung auf das in der Bundesrepublik zur Verfügung stehende Konsumgüterangebot fest. Dies verarbeitete die Mehrheit der DDR-Bevölkerung zu der Vorstellung, beim Massenkonsum mit einer nicht allzu großen Verzögerung doch noch da anzukommen, wo man sich im Westen bereits befand.

Das auf dieser Sicht der Dinge beruhende Gefühl der Übereinstimmung weiter Kreise der DDR-Bevölkerung mit der (Konsum-)Politik der SED-Führung erlitt jedoch Anfang der 80er Jahre einen gewaltigen Schlag, als deutlich wurde, dass die Entwicklung des Konsumgüterangebots der DDR nicht mehr auf Nachbzw. Einholen des bundesdeutschen Konsumniveaus hinauslief, sondern zunehmend durch Stagnation und Zurückbleiben gekennzeichnet wurde.

\section{Das Scheitern des Konsummusters der DDR während der 80er Jahre}

Die Ursache für das die Bevölkerung enttäuschende Konsumangebot ab Anfang der 80er Jahre war nicht auf eine Wende in der Haltung der SED zum Massenkonsum zurückzuführen. Offiziell galt die Strategie der „Einheit von Wirtschafts- und Sozialpolitik" weiter und wurde von Honecker auf jedem SED-Parteitag erneut beschworen. Die Ursache lag vielmehr im Scheitern der importinduzierten Modernisierungsstrategie. Auf Grund einer Reihe von Faktoren, die teilweise von der SED-Führung verschuldet waren wie die nachlassenden eigenen Innovationsanstrengungen, wie auch von Faktoren, die sie nicht zu verantworten hatte, wie die negativen Folgen der Erdölschocks von 1973 und 1979 für die Weltwirtschaft, blieben die Anfang der 1970er Jahre erhofften hohen Devisenerlöse aus. Die Devisenverschuldung nahm deshalb rasch zu. Das negative Handelsbilanzsaldo war der Hauptgrund, warum die Verschuldung der DDR gegenüber dem westlichen Ausland von 1975 bis 1980 dramatisch von $8,8 \mathrm{Mrd}$. VM (Valutamark: $1 \mathrm{VM}$ entsprach etwa $1 \mathrm{DM}$ ) auf 23,6 Mrd. VM angestiegen war (Bundesbank: 60).

Das Schuldenproblem wurde zunächst von der SED-Führung klein geredet. Nachdem aber Anfang der 80er Jahren die Zahlungsschwierigkeiten anderer realsozialistischer Länder wie Polen, Rumänien bzw. Jugoslawien bekannt wurden, sank die Kreditwürdigkeit der DDR auf dem Weltfinanzmarkt zeitweise ins „Bodenlose“. Jetzt erst war die SED-Führung bereit, ihre Außenfinanzen in Ordnung zu bringen. Waren die Depositen der DDR auf westlichen Banken 1981 noch kräftig gesunken, so gelang es 1982 sie auf 1,3 Mrd. Dollar zu erhöhen. Für dieses „Depositenwunder“ entscheidend war eine Erhöhung der Exporte der DDR in den Westen um 6\% gegenüber dem Vorjahr bei gleichzeitiger Re- 
duzierung der Importe aus den Hartwährungsländern um 15\%. Das ergab einen Devisenzuwachs von 4,7 Mrd. VM (Bundesbank: 53f). „Es war wirklich bemerkenswert“, urteilt auch die Leiterin der Abteilung „DDR und östliche Industrieländer“ des DIW, Doris Cornelsen, „wie diese Planwirtschaft es geschafft hat, das Defizit im Westhandel zu senken und sogar in einen Überschuss zu verwandeln sowie eine Schuldenkrise zu verhindern." (Anatomie: 21).

Das „Depositenwunder“ hatte allerdings seinen Preis - für die Wirtschaft der DDR generell und speziell auch für die weitere Entwicklung des Massenkonsums in der DDR. Der bestand nicht nur in einer drastischen Kürzung der Direktimporte von Konsumgütern aus dem Westen. Der bestand nicht nur darin, dass bestimmte heimische hochwertige und damit weltmarktfähige Konsumgüter kaum noch in DDR-Läden angeboten wurden. Der bestand vor allem darin, dass die Konsumgüterproduktion bei der Verteilung der Investitionen auf die Industriezweige stark vernachlässigt wurde.

Die Folgen für das Konsumgüterangebot waren erstens generell geringere Produktivitätsfortschritte und eine verminderter Stückzahl industrieller Konsumgüter im Angebot. Deutlich hinter der Nachfrage zurück blieben nicht nur die für lange Wartelisten notorisch bekannten PKW (Saretzki u. a.: 148f), sondern z.B. auch Geschirrspülmaschinen oder Farbfernseher. Investitionseinschränkungen im Konsumgütersektor bedeuteten zweitens den Verzicht auf den Aufbzw. Ausbau von Kapazitäten für neue Erzeugnisse, die in den 80er Jahren den Massenkonsum in der Bundesrepublik bereits mitbestimmten. Die Produktion solcher Novitäten wie Mikrowellengeräte, elektrische Wäschetrockner, Sofortbildkameras, Videorecorder, Heimcomputer und CD-Player wurde in der DDR nicht mehr oder nur noch in kleiner Stückzahl aufgenommen (Schwartau/Vortmann 1989: 300). Gleichzeitig konnten die DDR-Bürger diese elektrischen bzw. elektronischen Haushalts- und Freizeitgeräte nicht nur in der Werbung des Westfernsehens bewundern, sondern sie immer häufiger auch bei ihren Verwandten in der Bundesrepublik, die sie in den 80er Jahren in größerem Maßstabe als je nach 1961 besuchen durften, in Aktion sehen (Materialien: 529). ${ }^{3}$ Drittens existierten auch bei denjenigen industriellen Konsumgütern, bei denen sich die Ausstattungsrade in ost- und westdeutschen Haushalten nur geringfügig unterschied oder gleich war, Qualitäts- und Sortimentsunterschiede zuungunsten der DDR. Gemessen am Niveau der Bundesrepublik dominierten in der DDR Erzeugnisse unterer bis mittlerer Qualität. Die Gestaltung entsprach nicht dem modernen Stand. Die Sortimentsbreite der angebotenen Nahrungs- wie der übrigen Konsumgüter war eng begrenzt, die Verpackung primitiv. Textilien, Bekleidung und Schuhe entsprachen in ihrer

3 Im Jahre 1983 verfügten 24\% aller Haushalte in der Bundesrepublik über eine Geschirrspülmaschine, 15\% über elektrische Bügelmaschinen, 10\% über elektrische Wäschetrockner und 7\% über Videorecorder. 1985 verfügten erst 38\% der DDR-Haushalte über einen Farbfernseher, in der Bundesrepublik 1983 bereits 73\%. 
modischen Aktualität häufig nicht den Käuferwünschen (Bundesministerium 1987: 528).

Die Unterschiede zwischen dem von der SED-Führung verkündeten Ideal - es galt offiziell weiterhin die Strategie der „Einheit von Wirtschaft- und Sozialpolitik" und der Wirklichkeit traten in den 80er Jahren im Bereich des Konsums auf eine sehr unmittelbare und sinnlich erfahrbare Weise hervor.

Löste bereits die wieder zunehmende Differenz in Umfang und Niveau des Massenkonsums zwischen beiden deutschen Gesellschaften bei der DDR-Bevölkerung Verärgerung und Unzufriedenheit aus, so erst recht die Verletzung der in der DDR gepflegten Gleichheitsvorstellungen im Einkommens- und Konsumbereich durch neue Züge in der Konsumpolitik der DDR-Regierung. Vorstellungen über Verteilungsgerechtigkeit bis hin zur sozialen Nivellierung waren in der DDR-Geschichte über lange Jahre jener Kitt gewesen, der die Herrschenden und die Beherrschten zusammengehalten hatte. Es ist angesichts dessen schwer nachzuvollziehen, dass sich SED-Führung in einer Situation, in der sie über das Konsumniveau selbst kaum noch Zustimmung für ihre Politik bei der Bevölkerung einheimsen konnte, entschloss, in der Praxis auch von den aus der kommunistischen Utopie abgeleiteten Gleichheitsvorstellungen Abschied zu nehmen. Diesem Schritt lag jedoch kein Sinneswandel in der SED-Führungsspitze zugrunde - persönlich hingen die alternden Politbüromitglieder durchaus weiterhin den Konsum- und den Gleichheitsvorstelllungen der Arbeiterklasse aus den 1920er bzw. 1930er Jahren an. Das galt nicht unbedingt für ihren eigenen Lebensstil. Doch dürfte der sich nach dem, was darüber nach der „Wende“ bekannt geworden ist, zwar von dem Massenkonsum der Arbeiter, nicht aber wesentlich von dem der wohlhabenden Handwerker und der Gewerbetreibenden in der DDR unterschieden haben (Merkel 2004: 16). ${ }^{4}$ Vielmehr waren die zu einer stärkeren Differenzierung des Konsums führenden Politbürobeschlüsse der gleichen Ursache geschuldet, die auch Fortschritt des Massenkonsums bremste - der Notwendigkeit der Entschuldung gegenüber dem Westen.

Zu Beginn der 80er Jahre wurden die „Intershop-Läden“, die bis dahin nur an Flughäfen, Autobahnraststätten bzw. in Hotels überwiegend Konsumgüter westlicher Provenienz gegen Devisen an Westdeutsche und Ausländer verkauft hatten, für DDR-Bürger, die über „Westkontakte“ verfügten, zugänglich. Die Regierenden gingen berechtigterweise davon aus, dass sich ein größerer Teil der DDR-Bevölkerung von Verwandten bzw. Bekannten aus der Bundesrepu-

4 In der DDR-Statistik ist die Gruppe der höheren Funktionäre in Partei- und Regierung nicht ausgewiesen und ihr Einkommen nicht im Nachhinein zu berechnen. Nach Angaben von Ina Merkel lag das Einkommen der Handwerker1980 beim 1,6 fachen, das von Gewerbetreibenden bzw. der Leiter der 1972 verstaatlichten Privatbetriebe beim 1,9 fachen des DDRDurchschnitts. Der Zugang der Spitzenfunktionäre zu „Westwaren“ entsprach in den 80er Jahren in etwa dem jenes Teils der Bevölkerung, der über Westkontakte verfügte. 
blik D-Mark beschaffen konnte. Das Netz dieser Intershopländern wurde zielgerichtet erweitert. Jeder der mehr als 200 Kreise der DDR sollte nunmehr mindestens über einen Intershopladen verfügen. 1988 existierten annähernd 1000 Intershop-Verkaufsstellen, die einen jährlichen Umsatz von rund 1,1 Mrd. DM tätigten (Saretzki u.a.: 150). Da die „Westwaren“, auch westdeutsche Markenwaren, zu einem beträchtlichen Teil auf dem Wege der „Gestattungsproduktion" in DDR-Betrieben hergestellt wurden", eröffnete sich für die durch Zins- und Tilgungsforderungen westlicher Banken bedrängte DDRRegierung neben den Exportüberschüssen mit den Intershop-Verkaufsstellen eine zusätzliche Möglichkeit der Deviseneinnahme.

Dem größeren Teil der DDR-Bevölkerung, der nicht über die entsprechenden „Westkontakte“ verfügte, blieb allerdings die Möglichkeit verwehrt, über den "Intershop“ an das gehobene Konsumangebot heranzukommen. Die dadurch bewirkte Spaltung in Konsumenten erster und zweiter Klasse, ist von der SEDFührung zunächst in Kauf genommen worden. Die aus der Verletzung des Gleichheits- - indirekt auch des Leistungsprinzips - resultierende Unzufriedenheit in der DDR-Bevölkerung wurde dabei offensichtlich unterschätzt.

Unterschätzt wurden auch die negativen mentalen Auswirkungen der Konzentration der knappen Investitionsmittel in Betrieben mit „Westexportchancen“ auf das technische und Leitungspersonal der übrigen, dem Verschleiß ausgesetzten Industriebetriebe (Schwarz 2004: 184). ${ }^{6}$ Nicht einmal als Problem gesehen wurde von der SED-Führung, dass seit Mitte der 70er Jahre eine Jugend herangewachsen war, die - demographisch bedingt - nicht mehr, wie die Generation zuvor, ihre Frustration über die zunehmende Starrheit des politischen Systems unter Honecker durch Karrieren in Wissenschaft und Wirtschaft kompensieren konnte. „Fügsamkeit zugunsten von sozialer Sicherheit und mäßigem Fortkommen erschien [...] als zu hoher Preis, zumal ein Wandel der DDR, ein Abbau ihrer autoritären Strukturen, so wenig absehbar war wie ein wirtschaftlicher Aufschwung des Landes“"(Staritz 1996: 330).

Anders als bei den genannten Problemfeldern machte sich die SED Führung im Konsumbereich über Maßnahmen zur Schadensbegrenzung Gedanken. So entstand in den 1980er Jahren in der DDR ein umfassendes Netz von „Delikat"- und „Exquisit“-Verkaufsstellen. In den „Delikat-Läden“ gab es hochwertige Nahrungs- und Genussmittel, die zum Teil dem in den Intershops angebotenen Sortiment entsprachen. In den „Exquisit-Läden“ wurden vor allem

5 Streng ökonomisch genommen handelte es sich um einen Fall von Lohnveredlung. Zu den bekanntesten Marken gehörten Salamanderschuhe, Triumph-Bekleidung, Niveacreme sowie die Erzeugnisse von Adidas und Schießer.

6 In den Erinnerungen des Generaldirektors eines Bitterfelder Chemiekombinats heißt es dazu: „Das Knirschen und Vibrieren, die Abnutzungen und das Krankwerden der Anlage, die nicht zurückzudrängenden Verschmutzungen der Luft und der Abwässer beherrschten zunehmend unsere Arbeit". 
modische und qualitativ gute Bekleidung sowie Schuhe und Lederwaren verkauft. 1988 existierten in der DDR ca. 900 „Delikat“- und 1500 ExquisitVerkaufsstellen (Saretzki u.a. 150ff). Die Waren gehobenen Bedarfs wurden zwar in Mark der DDR, aber zu Preisen, die zwischen dem 5- und 9 fachen des DM-Preises (in den Intershop-Verkaufsstellen) lagen, angeboten. Nur die „Besserverdienenden“ in der DDR ohne DM-Bezug konnten sich regelmäßig über Delikat und Exquisit versorgen, so dass die Spaltung der Konsumenten keineswegs aufgehoben war.

\section{Das Ende der realsozialistischen Konsumgesellschaft}

Das Arrangement, das der DDR-Konsumkultur zugrunde lag und mit dem sich die SED-Führung während dreier Jahrzehnte die Zustimmung großer Teile der Bevölkerung erworben hatte, ging also im vierten Jahrzehnt in die Brüche. In den 80er Jahren gelang es der DDR-Regierung weder, das Tempo des Fortschritts in der Konsumgüterversorgung aufrecht zu erhalten, das bis dahin unter den Verbrauchern der DDR die Zuversicht, an das „Westniveau“ mit der Zeit schon heranzukommen, erzeugt hatte. Noch war die SED-Führung in der Lage, die aus der eigenen kommunistischen Utopie abgeleiteten und von der Bevölkerung vielfach geteilten Gleichheitsvorstellungen im Konsumbereich weiterhin zu verwirklichen. Je mehr sich der Zugang zur verheißungsvollen Konsumwelt des Westens für jene Mehrheit verschloss, die nicht über Westkontakte verfügte und ihn auch nicht über „Delikat“- und „Exquisitläden“ kompensieren konnte, desto mehr wurden aber auch jenem eingangs genannten Tauschgeschäft die Grundlagen entzogen, das wesentlich zur Legitimität der SED-Herrschaft beigetragen hatte. Verstärkt wurde die Unzufriedenheit durch weitere gravierende Probleme der DDR-Gesellschaft, die, seit Mitte der 1970er Jahre entstanden, in den 80er Jahren vergeblich einer Lösung harrten. Sie konnten in diesem Beitrag nur angedeutet werden.

Wenn aber mit der DDR die letztlich durch das bundesrepublikanische Vorbild bestimmte Konsumkultur nicht mehr erreichbar war, dann war die Aufkündigung der Loyalität gegenüber SED-Führung durch die Mehrheit der Bevölkerung ein logischer nächster Schritt. Das Konsumziel war dann ohne die SED-Führung bzw. gegen sie anzustreben. Die Verwirklichung dieses Zieles war individuell über die Flucht in den Westen - z.B. im Gefolge von Botschaftsbesetzungen, ab Sommer 1989 auch über die durchlässig gewordene ungarische Grenze möglich. Sie konnte aber auch kollektiv durch den Anschluss der DDR an die Bundesrepublik verwirklicht werden.

Über die außerordentlich große Rolle, die die Attraktivität der bundesrepublikanischen Konsumkultur für die Bereitschaft der Masse der DDR-Bevölkerung zum Anschluss an die Bundesrepublik spielte, gibt es viele Zeugnisse aus der Zeit nach der Öffnung der Mauer am 9. November 1989. „Jeder DDR-Bürger 
kennt Jacobs Kaffee, Milka-Schokolade, Adidas Turnschuhe und Levi’s Jeans“, meldete die „Welt“ das Ergebnis erster Befragungen genau einen Monat nach dem Mauerfall. Eine repräsentative Studie der Frankfurter A. C. Nielsen GmbH und des Leipziger Instituts für Marktforschung zum Markenbewusstsein in der DDR stellte drei Monate später fest, dass man dort „weit mehr Markenartikel kenne als die Bundesbürger“. Anders als in der Bundesrepublik genössen die Markenartikel jedoch noch „grenzenloses“ Ansehen (Humann 1990: 63, 169).

Die Bevölkerung Ostdeutschlands betrachtete die DM als Symbol dieser Konsumwelt. Sie entschied sich in den Wahlen vom März 1990 mehrheitlich für diejenigen Politiker, die ihnen eine Währungsunion so bald wie möglich versprachen. Bei der Entscheidung der Wähler spielte auch die Zuversicht eine Rolle, das sich mit der Übernahme der sozialen Marktwirtschaft der Bundesrepublik auch die anderen gravierenden Probleme der 80er Jahre lösen würden. Nicht umsonst hatte die CDU-dominierte „Allianz für Deutschland“ im Wahlkampf für jene, denen es nicht nur um eine materielle Besserstellung ging, die Losung: „Wählt uns, damit sich Leistung endlich lohnt“ ausgegeben (Zimmerling/Zimmerling: 234).

Die Erfüllung des Traums von westdeutsche Markenwaren für jedermann signalisierte die endgültige Ankunft der Ostdeutschen in der westlichen Konsumkultur. Dass sich diese nicht mit den Gleichheitsvorstellungen vertrug, denen die Mehrheit der DDR-Bürger während vier Jahrzehnten angehangen und denen sie noch einmal gefrönt hatten, als sie 1989/90 ihrer Empörung über die „Privilegien“ der SED-Führung Ausdruck gab, dass die Ostdeutschen mit der Ankunft in der Konsumkultur des Westens auch deren inneren Widersprüchen ausgesetzt wurde, erkannte die Mehrheit im Einheitsrausch, der für die letzten Monate der DDR charakteristisch war, zunächst nicht.

\section{Literatur}

Anatomie einer Pleite (2000): Der Niedergang der DDR-Wirtschaft seit 1971 (Materialien einer Diskussionsveranstaltung der Friedrich-Ebert-Stiftung), Berlin.

Bertsch, Georg C./ Matthias Dietz/Ernst Hedler (1990): SED: Schönes Einheitsdesign, Köln.

Bundesministerium für innerdeutsche Beziehungen (Hrsg.) (1987): Materialien zum Bericht zur Lage der Nation im geteilten Deutschland 1987, Bonn.

Cornelsen, Doris/Andreas Koch/Horst Lambrecht/Angela Scherzinger (1985): Konsumgüterversorgung in der DDR und Wechselwirkungen zum innerdeutschen Handel, Berlin.

Deutsche Bundesbank (1999): Die Zahlungsbilanz der ehemaligen DDR 1975 bis 1989, Frankfurt/M.

Deutsches Institut für Wirtschaftsforschung (DIW) (1977): Handbuch DDR-Wirtschaft, Reinbek.

Deutsches Modeinstitut (1960): Entwurf einer Vorlage zur Bestätigung des Modeschaffens in der DDR durch die Staatliche Plankommission, 25.3.1960 (Bundesarchiv, Bestand DDR, DE-1, 11968).

Falk, Waltraud /Horst Barthel (1966): Kleine Geschichte einer großen Bewegung. Zur Geschichte der Aktivisten- und Wettbewerbsbewegung in der Industrie der DDR, Berlin.

Geschichte der Sozialistischen Einheitspartei Deutschlands. Abriss (1978), Berlin.

Gesetz über den Siebenjahrplan zur Entwicklung der Volkswirtschaft der Deutschen Demokratischen Republik in den Jahren 1959 bis 1965 (1959), in: Der Siebenjahrplan des Friedens, des Wohlstands und des Glücks des Volkes, Berlin. 
Humann, Klaus, (Hrsg.) (1990): Wir sind das Geld. Wie die Westdeutschen die DDR aufkaufen, Reinbek.

Kaminsky, Anette (1999): Illustrierte Konsumgeschichte der DDR, Erfurt.

Merkel, Ina (2004): Alternative Rationalitäten, fremdartige Träume, absurde Utopien. Werbung, Marktforschung und Konsum im Sozialismus, in: Zeitgeschichte 1/2004

Mittelbach, Hans/Jörg Roesler (1989): Entwicklung von Einkommen und Verbrauch der Bevölkerung der DDR in den vergangenen vierzig Jahren, in: Jahrbuch für Soziologie und Sozialpolitik.

Roesler, Jörg (1988): Butter, Margarine und Wirtschaftspolitik. Zu den Bemühungen um die planmäßige Lenkung des Butter- und Margarineverbrauchs in der DDR zwischen 1950 und 1965, in: Jahrbuch für Wirtschaftsgeschichte 1/1988.

Roesler, Jörg (1995): Politik, Ökonomie und Industriedesign in Ostdeutschland, in: Deutschland Archiv 11/1995.

Roesler, Jörg (1997): Probleme des Brigadealltags. Arbeitsverhältnisse und Arbeitsklima in volkseigenen Betrieben 1950-1989, in: Aus Politik und Zeitgeschichte, B 38/97.

Saretzki, Hans-Ulrich/Reinhard Schenke/Günter Glende (1992): Von der Lebensmittelkarte zur Warteliste für das Auto - die planwirtschaftliche Entwicklung des Einzelhandels, in: Hölder, Egon (Hrsg.): Im Trabi durch die Zeit - 40 Jahre Leben in der DDR, Wiesbaden.

Schmiede, Rudi/Edwin Schudlich (1977): Die Entwicklung der Leistungsentlohnung in Deutschland. Eine historisch-theoretische Untersuchung zum Verhältnis von Lohn und Leistung unter kapitalistischen Produktionsbedingungen, Frankfurt a. M.

Schmidt, Hans-Walter (1994): Schaufenster des Ostens. Anmerkungen zur Konsumkultur der DDR, in: Deutschland Archiv 4/94.

Schwartau, Cord/Heinz Vortmann, (1989): Die materiellen Lebensbedingungen in der DDR, in: Weidenfeld, Werner/Hartmut Zimmermann (Hrsg.): Deutschland-Handbuch. Eine doppelte Bilanz 1949-1989, Bonn.

Schwarz, Heinz (2004): Prägungen aus acht Jahrzehnten. Bitterfelder Weg eines Generaldirektors, Schkeuditz.

Staritz, Dietrich (1996): Geschichte der DDR. Erweiterte Neuausgabe, Stuttgart.

Steiner, Andrè (2004): Von Plan zu Plan. Eine Wirtschaftsgeschichte der DDR, München.

Storbeck, Dietrich (1963), Flucht oder Wanderung? Eine Rückschau auf Motive, Folgen und Beurteilung der Bevölkerungsabwanderung aus Mitteldeutschland seit dem Kriege, in: Soziale Welt 14/1963.

Sywottek, Arnold (1993): Zwei Wege in die „Konsumgesellschaft“, in: Schildt, Axel/Arnold Sywottek (Hrsg.): Modernisierung im Wiederaufbau. Die westdeutsche Gesellschaft der 50er Jahre, Bonn.

Ulbricht, Walter (1959): Der Siebenjahrplan des Friedens, des Wohlstands und des Glücks des Volkes, Berlin

Vortmann, Heinz (1985): Geldeinkommen in der DDR von 1955 bis zu Beginn der achtziger Jahre, Berlin.

Wilczek, Annette (2004): Einkommen - Karriere - Versorgung. Das DDR-Kombinat und die Lebenslage seiner Beschäftigten, Berlin

Zimmerling, Zeno/Zimmerling, Sabine (1990): Neue Chronik DDR. Berichte, Fotos, Dokumente, 4.-5. Folge, Berlin. 


\section{Michael R. Redclift}

\section{Das Kaugummi: Geschmack, Raum und die 'Schattenländer'}

Nur wenige Produkte waren in der Geschichte des 20. Jahrhunderts so allgegenwärtig wie das Kaugummi und doch hat es kaum wissenschaftliche Aufmerksamkeit erregt. Das Kaugummi ist ein Beispiel für ein neues kommerzielles Produkt, das im 20. Jahrhundert in den USA Kultstatus erreichte, und von dort aus in den Rest der Welt 'exportiert' wurde. Es ist ein Beispiel für die Globalisierung von Geschmack. Im folgenden wird erstens der komplexe geopolitische Hintergrund der Kaugummiproduktion untersucht. Zweitens wird diskutiert, welchen Beitrag das Kaugummi zur Formierung neuer Identitäten geleistet hat, und zwar sowohl im Hinblick auf seine Konsumenten als auch auf seine (weitgehend unsichtbaren) Produzenten. Drittens wird auf die sich wandelnde Beziehung zwischen Moral und der Kommerzialisierung von Kaugummi eingegangen.

Die Geschichte des Kaugummis zeigt, dass hinter den hoch entwickelten Werbe- und Marketingstrategien, die vor allem die Anfänge der kommerziellen Kaugummiproduktion prägten, ein weiterer, fast unsichtbarer Prozess lag: der des chicle-Zapfens in Mexiko und der Kolonisierung der tropischen Wälder auf der mexikanischen Halbinsel Yucatan. Hier liegen die 'Schattenländer', die in der ersten Hälfte des 20. Jahrhunderts das Rohmaterial für den Massenkonsum des neuartigen Produkts lieferten. In der zweiten Hälfte des 20. Jahrhunderts trug die Entdeckung und Verbreitung des synthetischen Gummis dazu bei, dass sich das Kaugummi zu einem Kultobjekt und zu einer Ikone des Populärgeschmacks entwickelte.

\section{Das Kaugummi und die 'Schattenländer' des Konsums}

Der 'Boom' der chicle-Produktion, der auf die Befriedigung der nordamerikanischen Nachfrage ausgerichtet war, begann während der ersten zwei Jahrzehnte des 20. Jahrhunderts und erreichte seinen Höhepunkt in den frühen 1940er Jahren. Chicle, die Rohmasse aus der Kaugummi hergestellt wurde, wurde in Mittelamerika, vor allem auf der Halbinsel Yucatan gewonnen, wo in den hoch gelegenen, tropischen Wäldern der chicozapote-Baum wächst. Die Nachfrage nach chicle in den USA führte letztendlich zu einer Transformation der 